\title{
LOWER BODY NEGATIVE PRESSURE TRAINING LEADS TO LASTING REDUCTION IN CEREBRAL BLOOD FLOW: A PRELIMINARY STUDY USING ARTERIAL SPIN LABELING PERFUSION MRI
}

\author{
Stefan P. GAŹDZIŃSKI' ${ }^{1}$ Krzysztof KOWALCZUK², Piotr ZIELIŃSKI ${ }^{3}$, Paulina BARAN ${ }^{4}$, \\ Mariusz KREJ ${ }^{4}$, Łukasz DZIUDA ${ }^{4}$ \\ 1 Creative Neuroscience Lab - CNS Lab, Military Institute of Aviation Medicine, Warsaw, Poland \\ 2 Department of Simulator Studies and Aeromedical Training, Military Institute of Aviation Medicine, \\ Warsaw, Poland \\ 3 Department of Aviation Psychology, Military Institute of Aviation Medicine, Warsaw, Poland \\ 4 Department of Flight Simulator Innovations, Military Institute of Aviation Medicine, Warsaw, Poland
}

Source of support: This study was supported by the Polish National Centre for Research and Development within the framework of the Program for National Defense and Security under Project DOBR/0052/R/ID1/2012/03: (PI: Ł. Dziuda).

Author's address: Stefan P. Gaździński, Military Institute of Aviation Medicine, Krasińskiego 54/56 Street, 01-755 Warsaw, Poland, e-mail: stefan.gazdzinski@yahoo.com

Introduction: Human Centrifuge and the Lower Body Negative Pressure (LBNP) test are both used to diagnose abnormalities in responses of the cerebrovascular system to hypergravity. Though both methods lead to net displacement of blood towards lower extremities, they elicit different hemodynamic responses, as human centrifuge training involves significantly more muscle work, resulting in more blood being diverted to muscles during the test. Centrifuge training is also known to result in a long-term improvement of the cerebrovascular function. Therefore, it is not clear if both training procedures have the same short- and long-term effects on the cerebrovascular function.

Methods: Eight military cadet pilots ( $23.8 \pm 0.8$ years of age, one female) participated in the randomized, controlled, crossover study. To evaluate the differential effects of centrifuge training and LBNP on cerebral circulation post-training, we used pseudo-continuous arterial spin labeling (pCASL) 3T magnetic resonance imaging. The sequence was performed respectively before and after the LBNP training, as well as before and after the centrifuge training featuring equivalent protocols. The study was performed on two consecutive days. The values of mean CBF in gray matter were calculated and compared between conditions.

Figures: 5 • Tables 3 • References: 22 - Full-text PDF: http://www.pjambp.com • Copyright (c) 2018 Polish Aviation Medicine Society, ul. Krasińskiego 54/56, 01-755 Warsaw, license WIML •Indexation: Index Copernicus, Polish Ministry of Science and Higher Education 
Results: Centrifuge training did not affect the CBF. However, a 4.95\% $(p=0.048)$ decrease in CBF was recorded after equivalent LBNP training. Moreover, in the five participants who underwent LBNP training on the first day, the CBF remained depressed at $10.1 \%(p=0.042)$, however, the inter-participant variability was large.

Conclusions: The results should be considered preliminary, as only a small cohort participated in this study. Further studies are needed to investigate more in-depth and in larger cohorts the short- and long-term cardiovascular effects of centrifuge and LBNP trainings.

Keywords: MRI, arterial spin labeling, perfusion, cerebral blood flow, hypergravity, human centrifuge, lower body negative pressure (LBNP)

\section{INTRODUCTION}

Human Centrifuge and the Lower Body Negative Pressure (LBNP) test are used to diagnose abnormalities in responses of the cerebrovascular system to hypergravity. LBNP is a technique that redistributes blood from the upper body to the dependent regions of the pelvis and legs, thus reducing central venous pressure and venous return [6]. The major interest in LBNP began in the mid-1960s, when it was used to characterize the cardiovascular responses to hemorrhage and orthostatic stress, especially which associated with the weightlessness of space flight [6]. According to models, LBNP has no effect on flow rates in carotid, ulnar, and coronary arteries and the major effects are found in lower extremities [19]. However, LBNP leads to increased cardiac output, blood pooling in lower extremities and increased total peripheral resistance [12]. It was demonstrated that LBNP during $15^{\circ}$ head-down tilt mitigates elevation of intracranial pressure and increase of the cross-section of the internal jugular veins [20].

Human centrifuge, however, leads to acute reductions in cerebral blood flow (CBF) even at low acceleration values $[14,18]$. At higher acceleration values, the brain may be deprived of oxygen leading to a loss of consciousness, in aviation literature known as Gravity-induced Loss of Consciousness (G-LOC) [10]. At low acceleration values, increased systolic pressure, diastolic pressure and heart rate were recorded [4]. During centrifuge training, the participants generally strain muscles to prevent the blood from being displaced to lower extremities, this technique is called the Anti-G Straining Maneuvers (AGSM) $[3,16]$.

Although, both LBNP and human centrifuge lead to net displacement of blood towards lower extremities, they elicit different hemodynamic responses, as the centrifuge training involves signifi- cantly more muscle work, resulting in more blood being diverted to muscles during the test. Furthermore, it is generally assumed that both methods lead only to acute changes in hemodynamics.

The physical activity associated with both training procedures leads to acute reperfusion of the body and the central nervous system post-training. However, some effects of centrifuge training lasting more than a day were reported [21,22]. This raises a question whether both centrifuge training and LBNP training have hemodynamic effects that last minutes or hours after the training. Using pseudo-continuous Arterial Spin Labeling (ASL) for measuring Cerebral Blood Flow (CBF), we evaluated whether equivalent training procedures carried out on both devices lead to similar short-term (about 30 minutes) changes in brain hemodynamics and whether these potential changes last until the day following the training. ASL-CBF is a magnetic resonance imaging technique providing non-invasive access to CBF by magnetically labeling blood flowing to the brain $[1,13,17]$. ASL uses arterial blood water as an endogenous diffusible tracer by inverting the magnetization of the blood using radiofrequency (RF) pulses. After a delay allowing labeled blood to flow into the brain tissue, labeled images that contain signal from both labeled water and static tissue water are obtained. Separate control images without prior labeling of arterial spins are also obtained. The signal difference between control and labeled images provides a measure of labeled blood from arteries delivered to the tissue by perfusion [1].

The main task of the presented work was to assess the usefulness of the LBNP technique as a prescreening tool for initial candidates for aviation. The Aviation Academy mainly uses the centrifuge 
to check if the students showcase a correct cardiovascular reaction to accelerations. LBNP can be considered as a cheaper tool to eliminate candidates with disorders in this respect prior to centrifuge tests which are much more expensive compared to LBNP. The main idea was not to replace centrifuge tests with LBNP, as it is impossible due to differences in reactions of the body, but to supplement centrifuge tests with LBNP prescreening.

\section{MATERIALS AND METHODS}

\section{Participants}

This study was part of a larger project described in [8]. Of the larger cohort, who participated in a trail registered on 27/11/2017 at https://clinicaltrials.gov/ under number NCT0335423, eight military cadet pilots ( $23.8 \pm 0.8$ years of age, one female) took part in a study that evaluated cerebral hemodynamic responses to equivalent training procedures using LBNP and human centrifuge, performed on consecutive days in random order. All participants held current fitness to fly certificate issued by military and medical commission (i.e. they were healthy). The study was performed in the morning hours, after a light breakfast. The participants consumed no alcohol 24 hours prior to the study and refrained from caffeinated beverages during the study. On the first day, five participants took part in the LBNP training, whereas the remaining three participated in the centrifuge training. All participants were non-smokers, re- ported a to have slept well the night preceding the training and refrained from caffeinated beverages in the morning before the study.

A test-retest study evaluating the reproducibility of the MRI protocol was performed on a 45-year-old volunteer. The study protocol was approved in advance by the Bioethical Committee of the Military Institute of Aviation Medicine in Warsaw, Poland. Each participant provided their written informed consent before participating.

\section{Equipment}

For the purpose of the research described in this paper, we used the ORTHO-LBNP system by ETC-PZL Aerospace Industries, which consists of an LBNP chamber placed on a motorized tilt table to generate orthostatic (ORTHO) stress and thus strengthen the simulated stimuli. The system was developed by ETC-PZL Aerospace Industries to train military pilots in conditions of ischemic hypoxia and orthostatic hypotension. It is equipped with a number of measuring modules by the Institute of Medical Technology and Equipment, Poland, and the Nałęcz Institute of Biocybernetics and Biomedical Engineering Polish of the Academy of Sciences to monitor physiological parameters of a pilot during training. Therefore, research carried out using this system can become a source of new knowledge on counteracting the effects associated with ischemic hypoxia and orthostatic hypotension as well as minimizing the risk of their occurrence. The system is shown in Fig. 1 and was

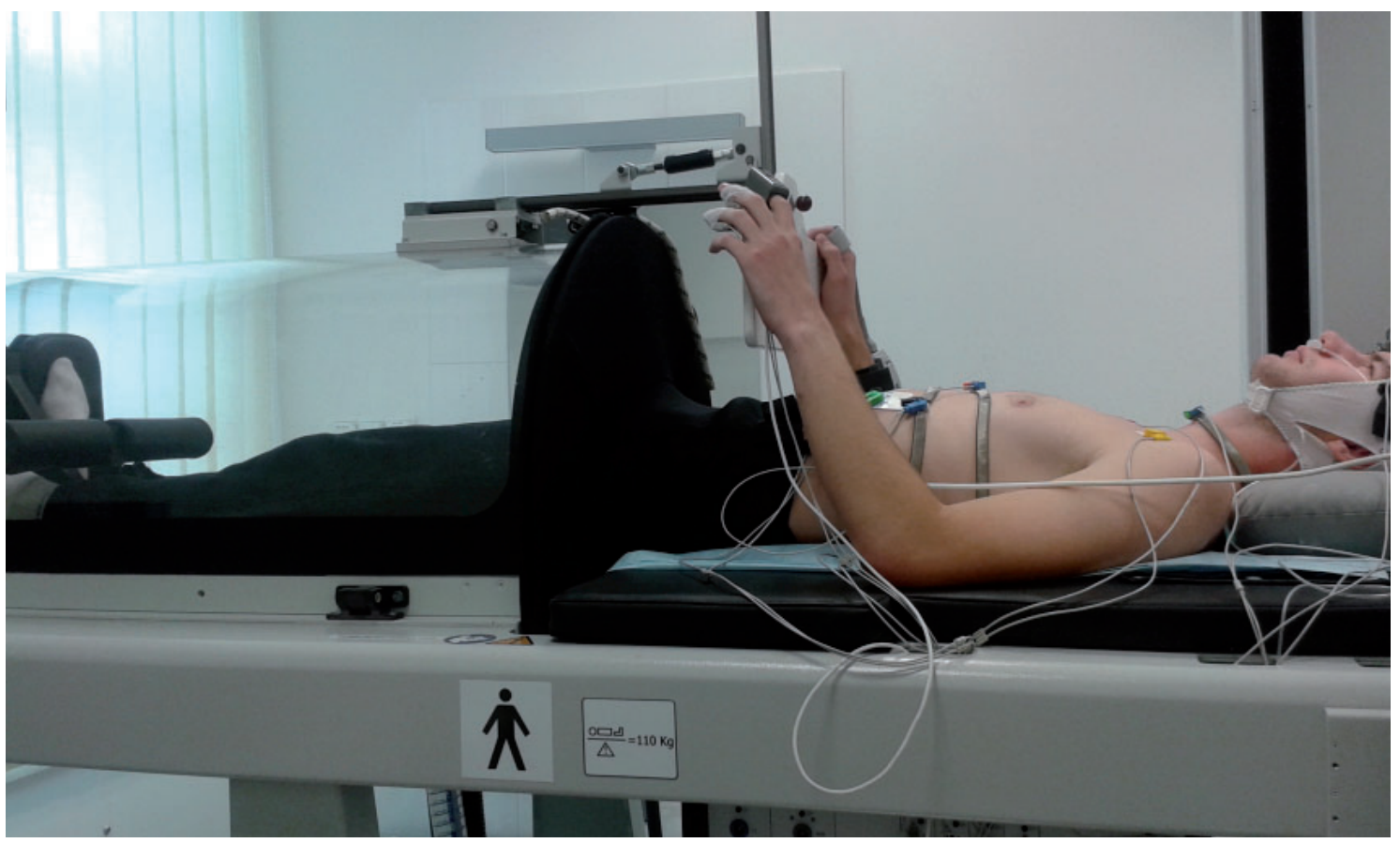

Fig. 1. A photograph of the ORTHO-LBNP system. 


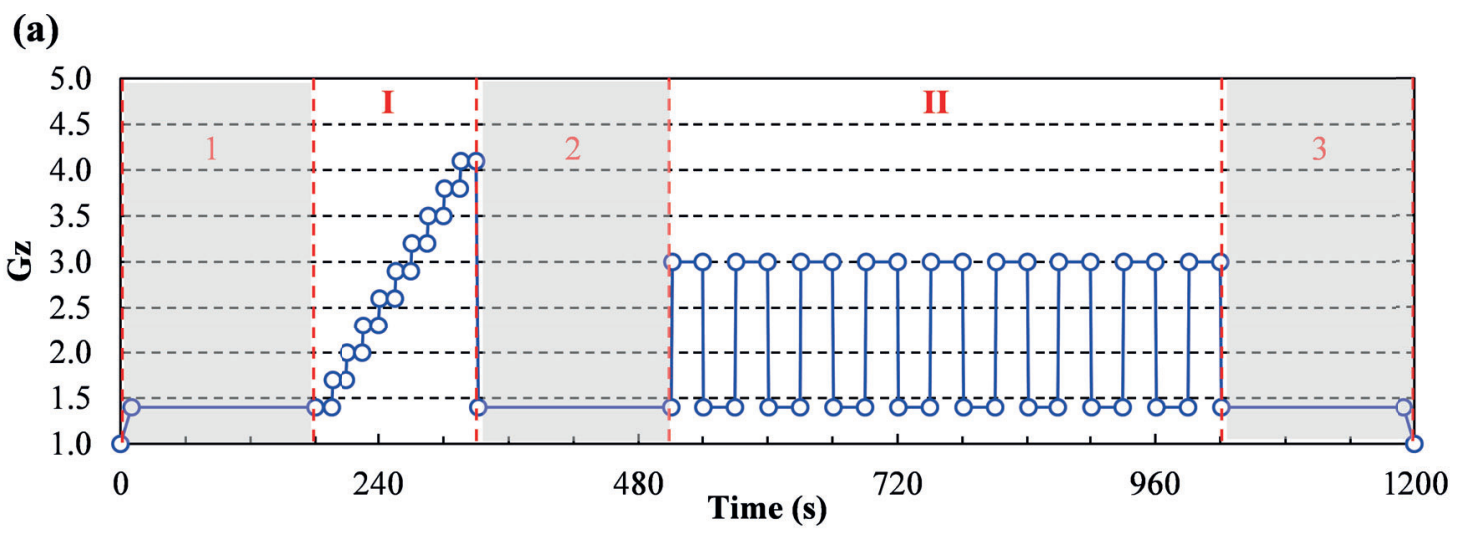

(b)

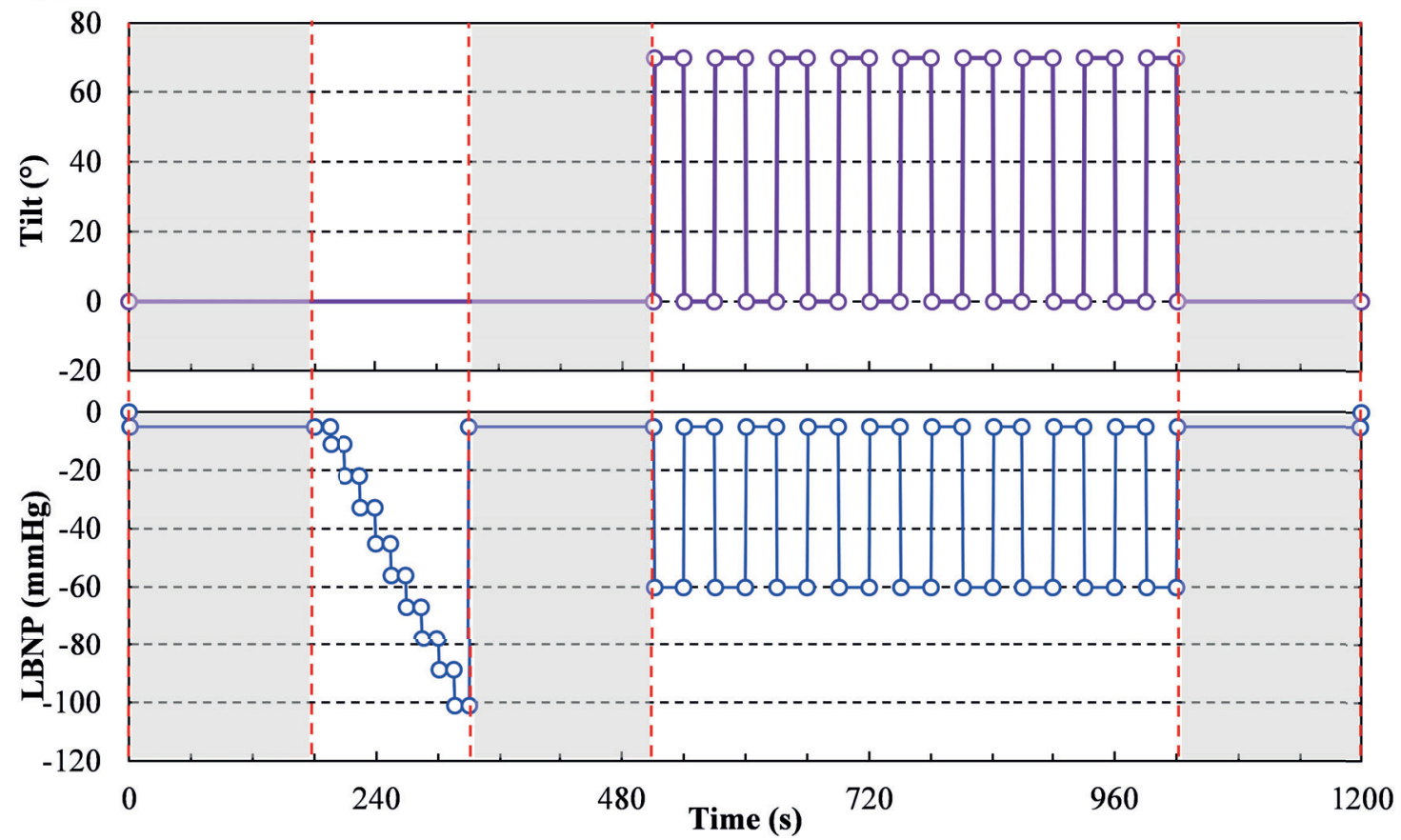

Fig. 2. Research protocols used in the study with the (a) centrifuge and (b) ORTHO-LBNP system. Gz is a multiple of Earth's acceleration of gravity.

described in detail in [16]. In this paper, 'ORTHOLBNP' also refers to the training method using orthostatic and LBNP stimuli.

HTC07 human centrifuge (AMST, Braunau, Austria: http://www.wiml.waw.pl/?q=en/Dynamic Flight_Simulator) was programmed to produce an equivalent series of accelerations $\mathrm{Gz}$ aimed to induce similar hemodynamic effects as the ORTHOLBNP system. The human centrifuge is a flight simulator that can also be used in operational, training and diagnostic profiles. It allows to carry out intensive pilot training, providing a chance to perform enhanced anti-G maneuvers and familiarize the aircrews with the effects of high accelerations and the push-pull phenomena. Moreover, the centrifuge provides a safe alternative allowing to raise awareness of aircrews in case of occur- rences of unwanted effects of accelerations, such as G-LOC or spatial disorientation. The gondola of the centrifuge is mounted on an 8-meter-long arm and allows to achieve Z-axis accelerations in the range from $-3 \mathrm{Gz}$ to $+16 \mathrm{Gz}$, with the maximal onset of accelerations of over $14,5 \mathrm{G} / \mathrm{s}$. Additionally, gyroscopic suspension of the gondola allows for achieving $\mathrm{X}$-axis and $\mathrm{Y}$-axis accelerations in the range of $\pm 10 \mathrm{G}$ and $\pm 6 \mathrm{G}$, respectively. Interchangeable parts of the centrifuge gondola allow for a functional projection of cockpit equipment of Polish Air Forces basic multi-purpose aircrafts, i.e. F-16 Block 52+ and MiG-29. The centrifuge is equipped with a number of devices to monitor the physiological effects induced on the pilot by accelerations. 


\section{Study protocols}

Two research protocols were used in the study, i.e., one for the experiment using the centrifuge and one for the experiment using the ORTHOLBNP system. As shown in fig. 2, each of them was composed of two stimuli phases to simulate (I) positive accelerations in the $z$-axis ( $+\mathrm{Gz}$ ) increased stepwise and (II) $+\mathrm{Gz}$ stimuli applied as an interval profile. Before and after the stimuli phases, there were resting phases, marked gray in fig. 1 as "1", "2" and " 3 ". Thus, the centrifuge protocol, as shown in fig 2. (a), consisted of five phases: (1) 180-second rest before the stimuli, (I) +Gz applied stepwise with $0.3 \mathrm{Gz} / 15 \mathrm{~s}$ decrement to $+4.1 \mathrm{Gz}$, (2) 180-second rest between the stimuli, (II) interval profile, i.e., $9 x+3 \mathrm{Gz}$ and $+1.4 \mathrm{Gz}$ applied alternately in 30-second intervals, and (3) 180-second rest after the stimuli. $+1.4 \mathrm{Gz}$ was achieved by the centrifuge moving at a minimum speed. The ORTHO-LBNP protocol was composed in the same manner. As shown in Fig. 2 (b), it consisted of five phases: (1) 180-second rest before the stimuli, (I) LBNP applied stepwise with $11.1 \mathrm{mmHg} / 15 \mathrm{~s}$ decrement to $-100 \mathrm{mmHg}$ (millimeter of mercury), (2) 180 -second rest between the stimuli, (II) interval profile, i.e., $9 \times 75^{\circ}$ head-up tilt (HUT) and accompanied by an exposure to an LBNP of $-60 \mathrm{mmHg}$, and (3) 180-second rest after the stimuli. The LBNP and tilt values were selected so that their impact on the body was similar to that observed for a given acceleration. According to the literature, supine LBNP at $-50 \mathrm{mmHg}$ should induce the hemodynamic response similar to that observed at an acceleration of $+2 \mathrm{Gz}$ [15]. Therefore, in the stepwise LBNP profile the maximum LBNP was set at -100 $\mathrm{mmHg}$ to simulate $+4 \mathrm{Gz}$ effects on heart rate (HR). In the interval profile, the maximum LBNP was set at $-60 \mathrm{mmHg}$ and HUT was additionally applied to simulate $+3 \mathrm{Gz}$ effects on HR. These values were adopted only for the purposes of the preliminary study. The proper choice of LBNP and tilt values is dependent on a number of parameters of the research system, e.g., the length of the LBNP chamber and the location of the sealing collar on the participant's body. The procedures for each of the research protocols took 20 minutes, excluding the preparation for the experiment and all the activities that were necessary after the experiment.

\section{MRI data acquisition}

All participants underwent CBF measurement using pseudo-continuous arterial spin labeling (pCASL; utilizing 3D Spiral Fast Spin Echo sequences, $\mathrm{TR} / \mathrm{TE} / \mathrm{TI}=4640 / 10.704 / 2025 \mathrm{~ms}, 1.875 \times 1.875$ $\mathrm{mm} 2$ in-plane resolution, $4 \mathrm{~mm}$ slice thickness, no gap, covering the entire brain, 50 averages, duration $4 \mathrm{~min} 22 \mathrm{sec}$ ) on a wide-bore 3T GE Discovery $750 \mathrm{w}$, using a body transmit coil for excitation and an eight-channel receive coil, within 15 minutes before and after both centrifuge and LBNP sessions.. This method is non-invasive and uses no exogenous radiotracers or contrast agents. Instead, arterial blood flowing to the brain is magnetically labeled at the neck prior the entry into the brain $[1,13,17]$ and allowed to travel to the brain tissue over the post-labeling delay time (TI) of 2025 ms. In this technique, water molecules in the blood flowing to the brain act as natural endogenous tracers to estimate brain tissue perfusion. CBF was calculated by the MRI scanner in milliliters of blood per $100 \mathrm{~g}$ of brain tissue per minute according to recommendations [1].

During the MRI scan, the participants were asked to keep their eyes open and rest quietly. They all confirmed adhering to the procedures after each session of the study. No participant reported conditions known to affect CBF: 1) no participant reported sleepiness during the study; sleep is associated with significant CBF decreases [2], 2) all participants refrained from caffeine the morning prior to the study and 3) all participants were non-smokers conditions known to affect CBF $[5,7,9]$. Exemplary $\mathrm{CBF}$ image is presented in fig. 3 .

A structural scan was performed using 3D IRPrepared Fast Spoiled Gradient Recalled Acquisition in Steady State (BRAVO) with the following parameters: TR/TI/TE $=8.484 / 3.268 / 450 \mathrm{~ms}, 1 \times 1 \times 1.2$ $\mathrm{mm}, 4 \mathrm{~min} 3 \mathrm{sec}$, covering the entire brain. These data were used to create voxel-based maps of probabilities of white matter, gray matter, and cerebrospinal fluid. Finally, T2-weighted images (TR/ $\mathrm{TE}=7338 / 98.112 \mathrm{~ms}, 0.4688 \times 0.4688 \mathrm{~mm}$ in-space resolution, $5 \mathrm{~mm}$ slice thickness, $0.5 \mathrm{~mm}$ gap) were recorded for co-registration between CBF and T1weighted images. Only the CBF sequences were recorded four times: before and after LBNP training, as well as before and after centrifuge training. The T1-w and T2-w images were recorded only once.

The participants were encouraged to talk about their experiences on the days preceding the studies to identify factors that could adversely affect the measurements, such as partying or alcohol consumption. No such incidents were identified. All procedures were performed in the morning hours.

\section{Data processing}

Global changes in gray matter CBF are evaluated in this paper. Post-processing steps are similar to previous reports [11] and depicted in fig. 4. 


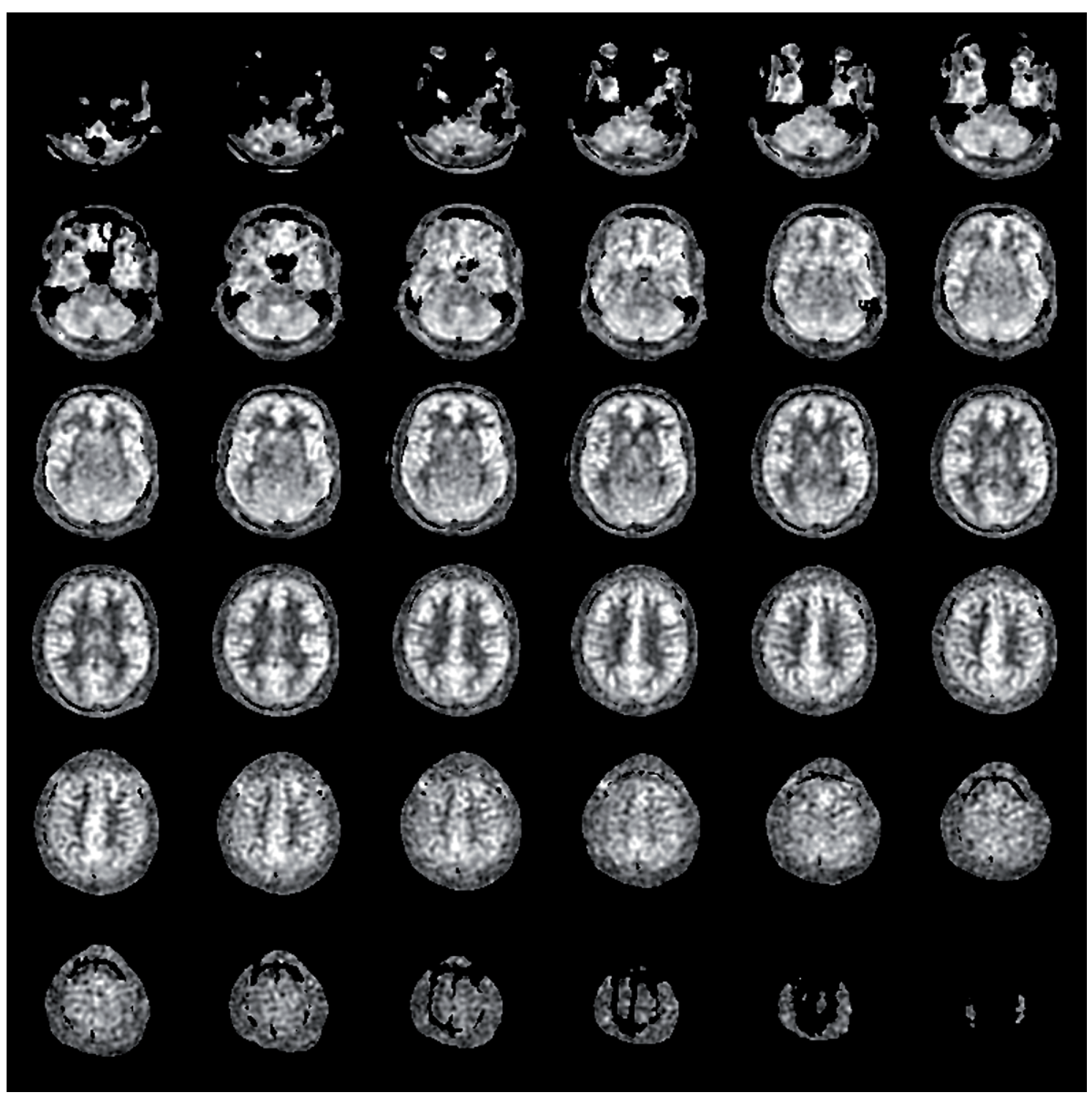

Fig. 3. Exemplary CBF image. Higher intensities depict higher CBF. Please note that CBF is higher in gray matter than in white matter.

Each untagged image (in the same space as the CBF image) was registered to the corresponding T1-w image via the T2-w image using SPM12 software (Wellcome Trust Centre for Neuroimaging, London, UK). The image quality was visually evaluated in each case. Probabilities of GM and WM in particular voxels of the T1-w were calculated via segmentation and then resampled to the CBF image space, thus creating a gray matter mask. Only CBF voxels containing at least $28 \%$ of gray matter were included into the analyses. No corrections for CSF contributions were made, as the study is longitudinal and each participant serves as their own control. Median CBF values for each participant were used in statistical analyses.

\section{Statistical Analysis}

Changes in (global, gray matter) CBF values were evaluated with paired t-tests. CBF changes over three time-points were evaluated using a repeated measures ANOVA. This procedure was implemented to for participants, who underwent their LBNP training on the first day, and for data obtained after the training, as well as the next day morning. The level of significance of $p<0.05$ was considered statistically significant. All statistical tests were conducted with $\mathrm{R}$ version 3.4.3 (with "ez" package written for that version of $\mathrm{R})$. 
$\mathrm{CBF}$

GM-mask
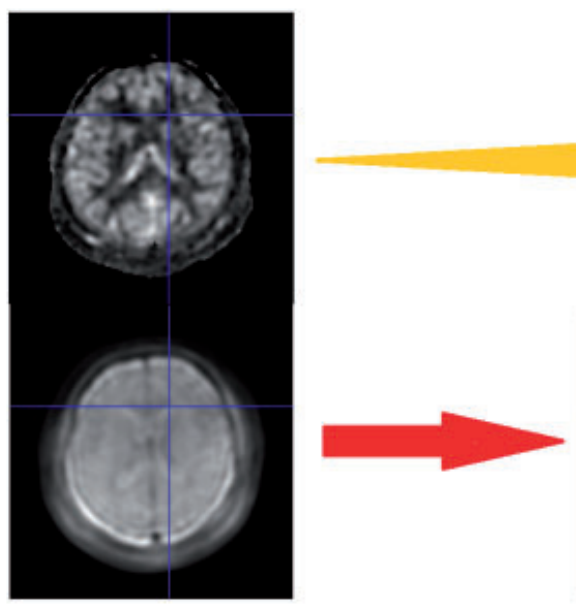

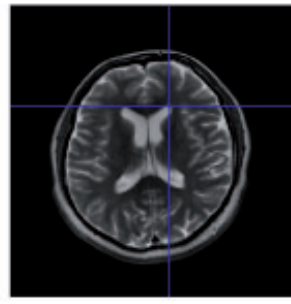

T2-w

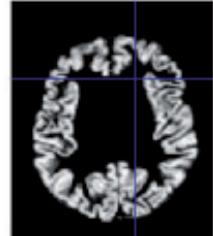

૫

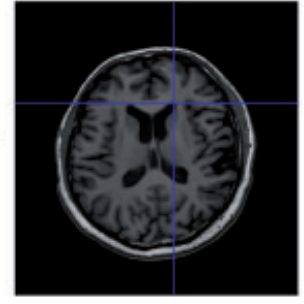

T1-w

untagged image

Fig. 4. CBF processing flow-chart. The CBF image is in the same orientation as the untagged image. As the latter image has more anatomical information than the CBF image, it was used for processing purposes. It was co-registered to the T1-w image via T2-w image. GM masks were obtained from segmented T1-w images and transformed to the CBF space.

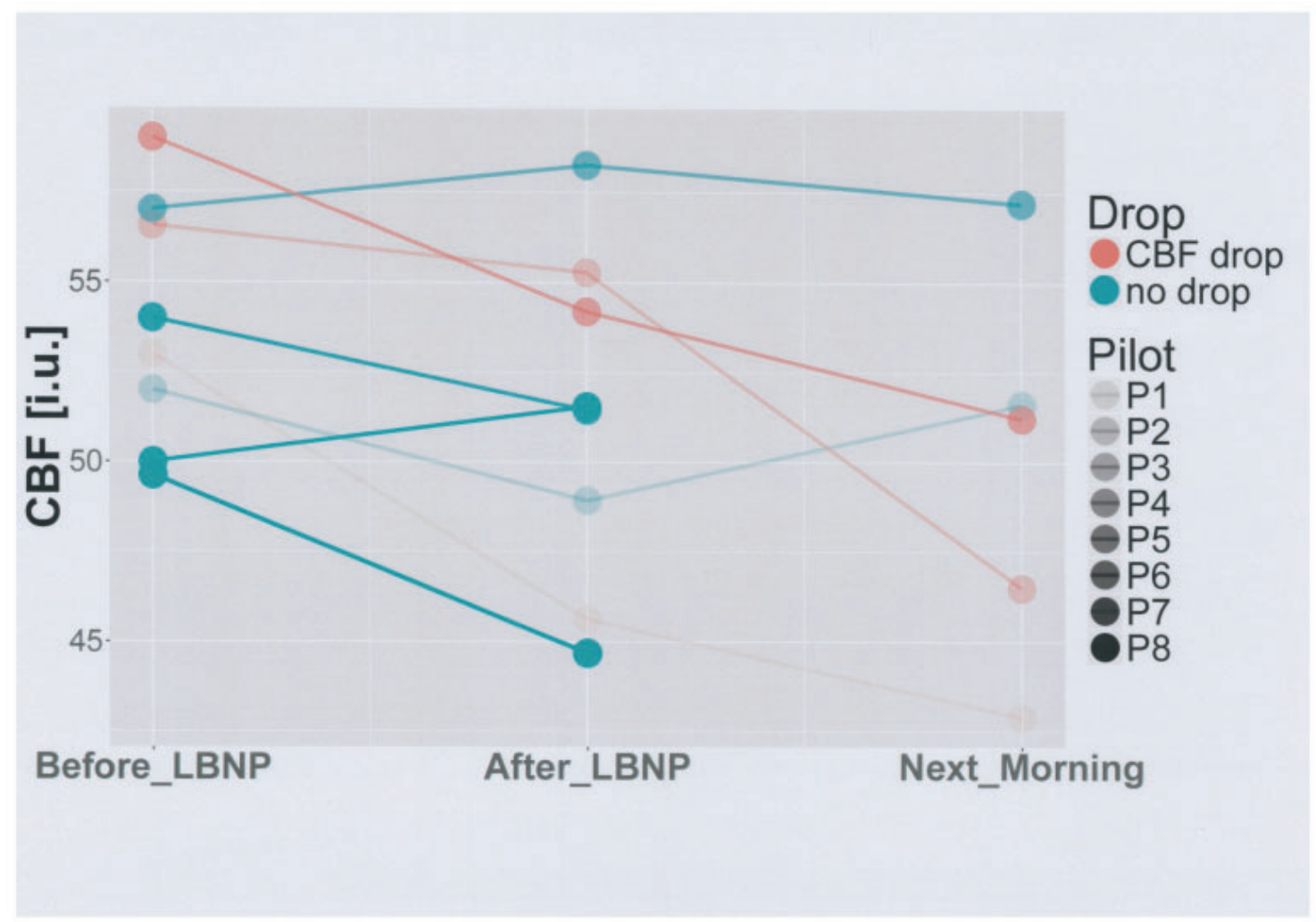

Fig. 5. Changes in mean CBF values before the ORTHO-LBNP training, thereafter, and the following day morning. 
Tab. 1. Mean values and changes in mean gray matter CBF induced by ORTHO-LBNP training. Please note the magnitude of CBF changes (last column). Subjects 1-5 took part in the LBNP training first, while subjects 6-8 participated in the centrifuge training first.

$\begin{array}{cc}\text { Participant } & \begin{array}{c}\text { CBF before ORTHO-LBNP training } \\ \text { [ml/100g/min] }\end{array} \\ 1 & 53.0 \\ 2 & 52.0 \\ 3 & 56.6 \\ 4 & 57.0 \\ 5 & 59.0 \\ 6 & 54.0 \\ 7 & 50.0 \\ 8 & 49.6 \\ \text { Mean } \pm \text { SD } & \mathbf{5 3 . 9 \pm 3 . 4}\end{array}$

CBF after ORTHO-LBNP training
[ml/100g/min]
45.6
48.9
55.3
58.2
54.2
51.5
51.6
44.7
$\mathbf{5 1 . 2} \pm \mathbf{4 . 7}$

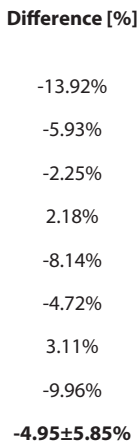

Tab. 2. Mean values and changes in mean gray matter CBF induced by the centrifuge training.

Participant
1
2
3
4
5
6
7
8

Mean \pm SD

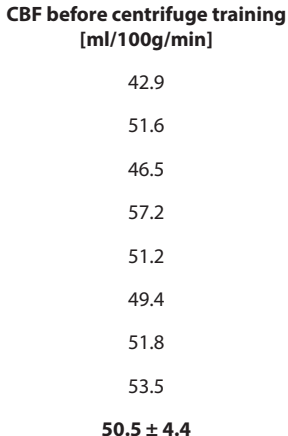

$50.5 \pm 4.4$

CBF after centrifuge training
[ml/100g/min]
42.9
53.3
49.0
56.7
51.7
49.7
47.4
53.0
$\mathbf{5 0 . 5} \pm \mathbf{4 . 2}$

Tab. 3. Individual CBF values on the day before the ORTHO-LBNP training and in the next-day morning, before the centrifuge training. Please note that the Table contains only data of participants who took part in the ORTHOLBNP training before the centrifuge training. Please also note that the mean drop in CBF is numerically higher than after the ORTHO-LBNP training.

$\begin{array}{cc}\text { Participant } & \begin{array}{c}\text { CBF before ORTHO-LBNP training } \\ \text { [ml/100g/min] }\end{array} \\ 1 & 53.0 \\ 2 & 52.0 \\ 3 & 56.6 \\ 4 & 57.0 \\ 5 & 59.0 \\ \text { Mean + SD } & \mathbf{5 5 . 5} \pm \mathbf{2 . 9}\end{array}$

\section{RESULTS}

The maximum difference in CBF on the test-retest in the 45-year-old participant was $5.86 \mathrm{ml} / 100 \mathrm{~g} / \mathrm{min}$, which amounts to an $11.7 \%$ difference. However, the average was $2.69 \mathrm{ml} / 100 \mathrm{~g} /$ min or $6.1 \%$ in relative differences.

The mean global gray matter CBF values for all participants before and after the LBNP training, as well as percent changes in $\mathrm{CBF}$, are presented in table 1, whereas the results before and after the equivalent centrifuge training are collected in table 2 .

$\begin{array}{cc}\begin{array}{c}\text { CBF in the morning after ORTHO- } \\ \text {-LBNP training [ml/100g/min] }\end{array} & \text { Difference [\%] } \\ 42.9 & -19.1 \% \\ 51.6 & -0.7 \% \\ 46.5 & -17.8 \% \\ 57.2 & 0.3 \% \\ 51.2 & -13.2 \% \\ \mathbf{4 9 . 9 \pm 5 . 5} & \mathbf{- 1 0 . 1} \pm \mathbf{9 . 3} \%\end{array}$

Centrifuge training did not affect CBF. Half an hour after the training, mean CBF values for the pilots remained unchanged, as compared to the respective mean values before the training $(t[7]=$ $0.085, \mathrm{p}=0.934$, difference mean $=0.062 \mathrm{ml} / 100 \mathrm{~g} /$ min, confidence interval 95\%: -1.67-1.79,). However, when the pilots were exposed to the ORTHOLBNP training, mean CBF values decreased $(\mathrm{t}[7]=$ 2.423, $p=0.046$, difference mean $=2.653 \mathrm{ml} / 100 \mathrm{~g} /$ min, confidence interval 95\%: 0.06-5.24). It corresponds to a $4.95 \%$ drop in the group mean CSF value. 
Moreover, in case of the five participants who did LBNP training on the first day, the CBF tended to decrease further, as compared to the baseline $(\mathrm{t}[4]=2.446, \mathrm{p}=0.071$, confidence interval 95\%: $-0.76-12.02$, mean of the differences $=5.632$ i.u.). It translates to a $10.1 \%$ drop in CBF, compared to the baseline.

As a follow-up analysis, a repeated measures ANOVA was calculated for all five participants, who underwent the LBNP training on the first day, and included CBF values measured before, after and the morning following the LBNP training. The effect of time tended to be significant $(F[2,8]=$ 4.368, $p=0.052$, Generalized Eta-Squared $=0.236$ ), which further confirms that ORTHO-LBNP results in a CBF decrease (see fig. 5).

\section{DISCUSSION}

We have compared the effects of two hypergravity training procedures equivalent in terms of cerebral blood flow overload in healthy military cadet pilots. To our knowledge, this is the first experiment of this kind. We have observed unexpected reduction in CBF following the LBNP training, but no changes in CBF following the centrifuge training. Surprisingly, in the sub-group who took part in the LBNP training on the first day, $60 \%$ of the participants had depressed CBF, between $13 \%$ and $19 \%$, whereas the remaining two participants reported no changes in CBF.

The mechanism of the observed drops is not known. It should be stressed that this phenomenon is clinically insignificant. The Human Centrifuge and the LBNP test are both used to diagnose abnormalities in cerebrovascular system responses to hypergravity. Although, both training procedures cause net displacement of blood towards lower extremities., they trigger different hemodynamic responses, as the centrifuge training involves significantly more muscle work, thus more blood is diverted to muscles during the test.

Observed CBF reductions are not clinically significant. However, they may have a subtle effect on cognitive performance and possibly, lower resistance to hypergravity, as they potentially dimin- ish (CBF) reserve under conditions of hypergravity and thus pose a threat to acrobats or military pilots.

The results should be considered preliminary. Only a small cohort participated in this study and we did not use a multi-inversion time (multi-TI) sequence to measure $C B F$, that would account for variability in blood arrival time and other complementary methods. However, CBF is relatively insensitive to arterial transit time [1], especially for post-labeling delay time of $2025 \mathrm{~ms}$, as used in our study. Nevertheless, we cannot exclude the possibility that the changes in blood velocity in arteries partially accounted for the results. Furthermore, the absolute values of CBF were calculated using certain standardized values of parameters needed to calculate the CBF maps, such as the longitudinal relaxation time of blood that could have been affected during the experiment. The observed reductions in CBF are not clinically significant. However, they may temporarily decrease resistance to high accelerations encountered by military pilots or astronauts sent to space. Given this possibility, this study should be repeated in a larger cohort of pilots and/or cadets using multi-TI magnetic resonance sequence and other complementary methods to assess cerebral hemodynamics should be performed before the ORTHO-LBNP training and several times thereafter to evaluate the long-term effects of the ORTHO-LBNP training on Cerebral Blood Flow.

\section{CONCLUSIONS}

Centrifuge training does not affect $\mathrm{CBF}$, whereas ORTHO-LBNP results in a small, clinically insignificant drop in CBF that does not seem to normalize the following day. These results should be treated as preliminary. Nevertheless, the study of changes in CBF in pilots is important, as it allows to assess the effectiveness of regulatory mechanisms of the cerebrovascular system in conditions of hypergravity. Therefore, the reported phenomenon should be re-evaluated by other studies using CBF sequence accounting for transit times, as well as other complementary methods.

\section{ACKNOWLEDGEMENTS}

We would like to thank Eng. Mariusz Walas, Eng. Marcin Strojek, and Robert Kilian for assistance in conducting the study, as well as Andrzej Gaździński for his assistance in data processing. 


\section{AUTHORS' DECLARATION:}

Study Design: Stefan P. Gaździński, Krzysztof Kowalczuk, Paulina Baran, Mariusz Krej, and Łukasz Dziuda Data Collection: Stefan P. Gaździński, Krzysztof Kowalczuk, Paulina Baran, Mariusz Krej, and Łukasz Dziuda Manuscript preparation: Stefan P. Gaździński, Krzysztof Kowalczuk, Piotr Zieliński, Paulina Baran, Mariusz Krej, and Łukasz Dziuda Funds Collection: Łukasz Dziuda. The Authors declare that there is no conflict of interest.

\section{REFERENCES}

1. Alsop DC, Detre JA, Golay X, Gunther M, Hendrikse J, Hernandez-Garcia L, et al. Recommended Implementation of Arterial Spin-Labeled Perfusion MRI for Clinical Applications: A Consensus of the ISMRM Perfusion Study Group and the European Consortium for ASL in Dementia. Magn Reson Med. 2015; 73(1):102-16.

2. Braun AR, Balkin TJ, Wesenten NJ, Carson RE, Varga M, Baldwin P, et al. Regional cerebral blood flow throughout the sleep-wake cycle. An H2(15)O PET study. Brain. 1997; 120 ( Pt 7):1173-97.

3. Buick F, Hartley J, Pecaric M. Maximum Intra-Thoracic Pressure With Anti-G Straining Maneuvers and Positive Pressure Breathing During +GZ. Aviat Space Environ Med. 1992; 63(8):670-7.

4. Caiozzo VJ, Rose-Gottron C, Baldwin KM, Cooper D, Adams G, Hicks J, et al. Hemodynamic and metabolic responses to hypergravity on a human-powered centrifuge. Aviat Space Environ Med. 2004; 75(2):101-8.

5. Clement P, Mutsaerts H, Václavů L, Ghariq E, Pizzini F, Smits M, et al. Variability of physiological brain perfusion in healthy subjects - A systematic review of modifiers. Considerations for multi-center ASL studies. J Cereb Blood Flow Metab. 2017.

6. Crystal GJ, Salem MR. Lower Body Negative Pressure: Historical Perspective, Research Findings, and Clinical Applications. Journal of Anesthesia History. 2015; 1(2):45-54.

7. Domino EF, Ni L, Xu Y, Koeppe RA, Guthrie S, Zubieta JK. Regional cerebral blood flow and plasma nicotine after smoking tobacco cigarettes. Prog Neuropsychopharmacol Biol Psychiatry. 2004; 28(2):319-27.

8. Dziuda L, Krej M, Smietanowski M, Sobotnicki A, Sobiech M, Kwasny P, et al. Development and evaluation of a novel system for inducing orthostatic challenge by tilt tests and lower body negative pressure. Sci Rep. 2018; 8.

9. Field AS, Laurienti PJ, Yen YF, Burdette JH, Moody DM. Dietary caffeine consumption and withdrawal: confounding variables in quantitative cerebral perfusion studies? Radiology. 2003; 227(1):129-35.

10. Forster EM, Cammarota JP. The Effect of G-loc on Psychomotor Performance and Behavior. Aviat Space Environ Med. 1993; 64(2):132-8.

11. Gazdzinski S, Durazzo TC, Jahng GH, Ezekiel F, Banys P, Meyerhoff DJ. Effects of chronic alcohol dependence and chronic cigarette smoking on cerebral perfusion: A preliminary magnetic resonance study. Alcoholism-Clinical and Experimental Research. 2006; 30(6):947-58.

12. Hachiya T, Hashimoto I, Saito M, Blaber AP. Peripheral Vascular Responses of Men and Women to LBNP. Aviat Space Environ Med. 2012; 83(2):118-24.

13. Haller S, Zaharchuk G, Thomas DL, Lovblad KO, Barkhof F, Golay X. Arterial Spin Labeling Perfusion of the Brain: Emerging Clinical Applications. Radiology. 2016; 281(2):337-56.

14. Iwasaki K-I, Ogawa Y, Aoki K, Yanagida R. Cerebral circulation during mild plus Gz hypergravity by short-arm human centrifuge. J Appl Physiol. 2012; 112(2):266-71.

15. Lategola MT, Lyne PJ, Burr MJ. Cardiorespiratory Assessment of 24-hour Cras-Diet Effects on Altitude, +GZ, and Fatigue Tolerances. Aviat Space Environ Med. 1982; 53(3):201-9.

16. Macdougall JD, Mckelvie RS, Moroz DE, Moroz JS, Buick F. The Effects of Variations in the Anti-G Straining Maneuver on Blood-Pressure at +GZ Acceleration. Aviat Space Environ Med. 1993; 64(2):126-31.

17. Nery F, Gordon I, Thomas DL. Non-Invasive Renal Perfusion Imaging Using Arterial Spin Labeling MRI: Challenges and Opportunities. Diagnostics. 2018; 8(1).

18. Ogawa Y, Yanagida R, Ueda K, Aoki K, Iwasaki K-I. The relationship between widespread changes in gravity and cerebral blood flow. Environ Health Prev Med. 2016; 21(4):186-92.

19. Sud VK, Srinivasan R, Charles JB, Bungo MW. Effects of Lower-Body Negative-Pressure on Blood-Flow with Applications to the Human Cardiovascular-System. Med Biol Eng Comput. 1993; 31(6):569-75. 
20. Watkins W, Hargens AR, Seidl S, Clary EM, Macias BR. Lower-body negative pressure decreases noninvasively measured intracranial pressure and internal jugular vein cross-sectional area during head-down tilt. J Appl Physiol. 2017; 123(1):260-6.

21. Yang CB, Wang YC, Gao Y, Geng J, Wu YH, Zhang Y, et al. Artificial gravity with ergometric exercise preserves the cardiac, but not cerebrovascular, functions during 4 days of head-down bed rest. Cytokine. 2011; 56(3):648-55.

22. Yang $\mathrm{CB}$, Zhang S, Zhang Y, Wang B, Yao YJ, Wang YC, et al. Combined short-arm centrifuge and aerobic exercise training improves cardiovascular function and physical working capacity in humans. Med Sci Monit. 2010; 16(12):CR575-CR83.

Cite this article as: Gaździński SP, Kowalczuk K, Zieliński P, Baran P, Krej M, Dziuda Ł. Lower Body Negative Pressure Training Leads To Lasting Reduction In Cerebral Blood Flow: A Preliminary Study Using Arterial Spin Labeling Perfusion Mri. Pol J Aviat Med Bioeng Psychol 2018; 24(4):36-46. DOI: 10.13174/pjambp.25.05.2020.04 